\title{
Effect of chitosan coating combined with hypotaurine on the quality of shrimp (Litopenaeus vannamei) during storage
}

\author{
Meiyu Chen ${ }^{1,2}$, Lingping $\mathrm{Hu}^{1,2}$, Zhiheng $\mathrm{Hu}^{1,2}$, Yaqi Zhou ${ }^{1,2}$, Gaoshang Li, ${ }^{1,2}$, Yaoxian Chin ${ }^{1}$, Yaqin Hu ${ }^{1, *}$ \\ ${ }^{1}$ College of Food Science and Engineering, Hainan Tropical Ocean University, Sanya 572022, China \\ ${ }^{2}$ College of Biosystems Engineering and Food Science, National-Local Joint Engineering Laboratory of Intelligent Food Technology and Equipment, \\ Zhejiang Key Laboratory for Agro-Food Processing, Integrated Research Base of Southern Fruit and Vegetable Preservation Technology, Fuli Institute \\ of Food Science, Zhejiang University, Hangzhou 310058, China
}

\begin{abstract}
This study aimed to investigate the effect of different coating materials on the quality of shrimp (Litopenaeus vannamei) during chilled storage for 10 days. Fresh shrimp were randomly divided into five groups: the control group, the hypotaurine treatment group (2\%), the chitosan group (1\%), the hypotaurine + chitosan group ( $2 \%$ hypotaurine solution with $1 \%$ of chitosan), and the sodium metabisulfite treatment group (1.25\%). Compared with other treatments, the lower accumulation of total visible counts (TVC, 5.25 Log $_{10}$ CFU/g), total volatile basic nitrogen (TVB-N, $22.5 \mathrm{mg} / 100 \mathrm{~g}$ ) and thiobarbituric acid values (TBA, $0.58 \mathrm{mg} \mathrm{MDA}$ / $\mathrm{kg}$ ) suggested that coating of chitosan-hypotaurine could retard the microbial activity, protein degradation and lipid oxidation of shrimp. Meanwhile, results demonstrated that the chitosan coating combined with hypotaurine showed an excellent performance in inhibiting quality deterioration ( $\mathrm{pH} 7.5, \Delta \mathrm{E} 7.0$, hardness $393 \mathrm{~g}$, and elasticity 0.69). Furthermore, the melanosis degree of shrimp was alleviated, and the sensory parameters, including appearance, odor and texture, were maintained to the acceptable level by chitosan based hypotaurine treatment during the chilled storage.
\end{abstract}

Keywords: Litopenaeus vannamei, Chitosan coating, Hypotaurine, Chilled storage, Quality evaluation

\section{Introduction}

Litopenaeus vannamei, as one of the most common aquatic food resources in the world, holds a large market share and high nutritional values (Sánchez-Muros et al., 2020). However, the presence of black spots and microbial spoilage in improper storage conditions usually limited their expected shelf-life. In order to delay melanosis and prolong the shelf-life of perishable crustaceans, several anti-melanosis agents, for example, 4-hexylresorcinol, sulfite-based compounds and phosphates, have been studied for melanosis inhibition (Amparyup et al., 2013). Moreover, due to the potential hazards of synthetic chemical

Received: Oct 15, 2021 Revised: Dec 4, 2021 Accepted: Dec 30, 2021

${ }^{*}$ Corresponding author: Yaqin $\mathrm{Hu}$

College of Food Science and Technology, Hainan Tropical Ocean University, Sanya 572022, China

Tel: +86-15868109010, E-mail: 1004346262@qq.com

This is an Open Access article distributed under the terms of the Creative Commons Attribution Non-Commercial License (http://creativecommons.org/licenses/by$\mathrm{nc} / 4.0 /$ ) which permits unrestricted non-commercial use, distribution, and reproduction in any medium, provided the original work is properly cited.

Copyright $\odot 2022$ The Korean Society of Fisheries and Aquatic Science 
additives to human health, natural products, especially natural antioxidants and melanin inhibitors, have been intensively studied as alternatives (Sharifian et al., 2019; Shiekh et al., 2019).

Chitosan, a potential natural food preservative, has been widely used in seafood preservation due to its biodegradability, biocompatibility, remarkable antimicrobial and antifungal properties, and film-forming properties (Lim et al., 2021). Recent studies have shown that incorporation of natural preservatives in chitosan films significantly maintained the physicochemical quality of aquatic products and inhibited spoilage (Negm et al., 2020), thus extending the expected shelf-life during cold storage. Yuan et al. (2016) investigated the synergistic effects of chitosan and pomegranate extracts on the quality of Pacific white shrimp during cold storage, and demonstrated that simultaneous treatment with chitosan and pomegranate extracts exhibited more acceptable sensory properties and effectively delay the increase of total viable count (TVC) and total volatile base nitrogen (TVB-N) values than any other single treatments. Previous studies have shown that hypotaurine had an outstanding polyphenol oxidase inhibiting property and antioxidant property (Acharya \& Lau-Cam, 2013), and inhibited the formation of melanosis and maintained the quality of Litopenaeus vannamei during storage. It is an ideal alternative of sulfate compounds ( $\mathrm{Li}$ et al., 2020a).

In the aim of achieving better antibacterial effects and retaining the freshness of Litopenaeus vannamei, the conventional coating preservation method was used by immersing the shrimp in the chitosan/anti-melanosis agents and then drained in the air. The changes of color differences, melanosis degree, textural properties, $\mathrm{pH}, \mathrm{TVB}-\mathrm{N}$ and thiobarbituric acid values (TBA) values, TVC, and sensory properties during 10-days storage at $4{ }^{\circ} \mathrm{C}$ were carefully evaluated, and compared between different groups treated with/without hypotaurine, chitosan or sodium metabisulfite to investigate the synergistic effect of hypotaurine and chitosan on the quality of Litopenaeus vannamei during chilled storage.

\section{Materials and Methods}

\section{Materials}

Chitosan (CAS 9012-76-4), hypotaurine (CAS 300-84-5) and sodium metabisulfite (SMS, CAS 7681-57-4) were purchased from Sigma-Aldrich (Shanghai, China). Standard plate count agar (PCA), trichloroacetic acid (TCA), TBA, malondialdehyde, ethylenediamine tetraphosphate (EDTA), methyl red and ethyl cresol green were purchased from Sinopharm Chemical Reagent (Shanghai, China). The shrimp (Litopenaeus vannamei) were purchased from the local supermarket (Hangzhou, China).

\section{Sample preparation}

The shrimp (Litopenaeus vannamei) were randomly divided into 5 different treatment groups, including a control group, the hypotaurine treatment group, the chitosan group, combined with hypotaurine and chitosan treatment, and a SMS treatment. The concentration of the soaking solution of hypotaurine is $2 \%$ $(\mathrm{w} / \mathrm{v})$, of chitosan treatment is $1 \%(\mathrm{w} / \mathrm{v})$, of SMS treatment is $1.25 \%(\mathrm{w} / \mathrm{v})$. The shrimp were immersed into treatment solutions at a ratio of 1:2 (solution to shrimp) after $30 \min$ under $4^{\circ} \mathrm{C}$, and then taken it out and drain naturally at room temperature. The shrimp from different treatments were packaged again in sterile polyethylene bags and then stored at $4^{\circ} \mathrm{C}$ for further experiments.

\section{Evaluation of microbiological}

Microbial analyses were performed as described by Meiyu Chen (Chen et al., 2021). A total of $10 \mathrm{~g}$ samples were transferred into $90 \mathrm{~mL}$ of $0.09 \%(\mathrm{w} / \mathrm{v})$ sterilized sodium chloride $(\mathrm{NaCl})$ buffer solution and homogenized for $5 \mathrm{~min}$ using a stomacher blender at a low speed of 6 times per second. A 10-fold dilutions method was conducted by $\mathrm{NaCl}$ buffer solution before spreading and plating. The PCA was used as media for the TVC of bacteria test, and incubated at $37^{\circ} \mathrm{C}$ for $48 \mathrm{~h}$. the TVC results were expressed as $\log _{10} \mathrm{CFU}$ (colony - forming units) / g.

\section{$\mathrm{pH}$ value and $\mathrm{LAB}$ value}

During the storage, $\mathrm{pH}$ values of each sample were measured by a pH meter (Thermo Orion 710A+, Beverly, MA, USA) immediately in the suspension of tissue homogenates of $2 \mathrm{~g}$ minced shrimp and $20 \mathrm{~mL} 0.1 \mathrm{~mol} / \mathrm{L}$ potassium chloride solution.

Color changes of shrimp at different storage time were evaluated by a colorimeter (CR-10, Konica Minolta, Tokyo, Japan). The physical appearance of each shrimp sample was captured and the hunter color values were recorded. The total color difference $(\Delta \mathrm{E})$ was calculated as follows:

$$
\Delta E=\sqrt{\Delta \mathrm{E}^{2}+\Delta \mathrm{a}^{2}+\Delta \mathrm{b}^{2}}
$$

where $\Delta \mathrm{L}, \Delta \mathrm{a}$ and $\Delta \mathrm{b}$ represented the color difference between each shrimp after treatment to fresh (L, lightness; a, redness/greenness; b, yellowness/blueness). 


\section{Assay of texture profile analysis (TPA)}

The TA-XT2i Testing Machine (Stable-Micro System, Surrey, UK) was used to evaluate the texture of shrimp samples. The analysis is based on the compression of the ready-to-eat shrimp samples which imitates the human biting (Li et al., 2020b), with slight modification. The P5 flat-bottomed cylindrical probe, which is $50 \mathrm{~mm}$ in diameter, was selected to use. The texture measurement was composed of 2 consecutive compressions, and the measurement distance was $50 \%$ of the initial height of the shrimp sample. The descending speed and crosshead speed were all set to $1 \mathrm{~mm} / \mathrm{s}$ for $5 \mathrm{~s}$ under the $10 \mathrm{~g}$ trigger force. The values for hardness and elasticity are automatically calculated by the software, and each set of samples is measured in parallel 6 times.

\section{Assay of total volatile base nitrogen (TVB-N)}

TVB-N contents of each sample were determined according to the semi-micro steam distillation method of Ge et al. (2020). In brief, five grams of minced Litopenaeus vannamei shrimp were mixed with $25 \mathrm{~mL}$ of sterile deionized water. The mixture solution was homogenized by an oscillator (ZHWY-2102C, Shanghai Zhicheng Analytical Instrument Manufacturing, Shanghai, China) and shaken at $1,200 \times \mathrm{g}$ for $3 \mathrm{~h}$. Then, the homogenate was centrifuged at $16,000 \times \mathrm{g}$ for $10 \mathrm{~min}$ to get the supernatant. Subsequently, $5 \mathrm{~mL}$ of the supernatant was mixed with $5 \mathrm{~mL}$ of $10 \mathrm{~g} / \mathrm{L}$ magnesium oxide ( $\mathrm{MgO})$ suspension and analyzed using an automatic Kjeldahl Apparatus (KN780, Jinan Alva Instrument, Jinan, China). Results were described as mg/100 g.

\section{Assay of thiobarbituric acid (TBA)}

According to the method of Wu et al. (2016), $3 \mathrm{~g}$ of shrimp meat mixed with $30 \mathrm{~mL}$ of $7.5 \%(\mathrm{w} / \mathrm{v})$ TCA (containing $0.1 \%$ EDTA), and homogenized at $60,000 \times \mathrm{g}$ for $2 \mathrm{~min}$ and then centrifuged at $16,000 \times \mathrm{g}$ for $10 \mathrm{~min}$. The TBA solution $(0.02 \mathrm{~mol} / \mathrm{L})$ was added to the supernatant solution at a ratio of 1:1. The mixture solution was held in the water bath at $90{ }^{\circ} \mathrm{C}$ for $40 \mathrm{~min}$. The absorbance value of the sample was measured at $532 \mathrm{~nm}$ with distilled water as blank, while with 1,1,3,3-tetraethoxypropane as the standard curve. The result was expressed as the milligram of malondialdehyde contained in $1 \mathrm{~g}$ shrimp meat (mg MDA/ $\mathrm{kg})$.

\section{Melanosis evaluation}

The melanosis state of Litopenaeus vannamei shrimp was determined by five professional panelists as described by Montero et al. (2001), and made some modifications. On each coating treatment group, 10 shrimp were evaluated for black spots on the surface and head by visual inspection, and then scored the intensity using a 10-point descriptive scoring scale (ranging from $0=$ no melanosis in the sample to $10=$ extremely heavy melanosis). The result was collected as the means of the scores given by the panelists.

\section{Sensory evaluation}

Sensory scores were assayed according to the method by 10 professionally trained sensory panelists (five male and five females, aged from 19 to 45), modified from Li et al. (2013). Ten shrimp of each treatment group during chilled storage were evaluated from three aspects: appearance, odor and texture properties. Each panelist had completed $8 \mathrm{~h}$ of training to familiarize themselves with the attributes. The scoring range of each parameter is $0-10$ with radar chat, of which 10 is the strongest intensity of the attribute, while 0 denotes the lowest quality and the most unacceptable. The results presented as the radar charts are the average number from all sensory panelists.

\section{Statistical analysis}

All measurement steps were repeated 3 times, and parallel experiments were performed in triplicates. The experimental results were usually expressed in the form of mean \pm SD. The data were analyzed by ANOVA and Duncan multiple comparison ( $p$ $<0.05)$. The figures were drawn by origin 9.0 software.

\section{Results and Discussion}

\section{Analysis of total visible counts (TVC) change}

The effects of different coating treatments on the TVC of Litopenaeus vannamei during 10 days of iced storage are shown in Fig. 1. The TVC of all shrimp samples significantly $(p<0.05)$ increased according to the time of storage, but the increasing rates were different, from fastest to lowest was the control group, SMS group, hypotaurine group, chitosan group, and the hypotaurine - chitosan group, respectively. With the increase of storage time, the bacteria in the control group grew much higher than those in the coating treatment groups, indicating that all coating methods of the SMS, chitosan and hypotaurine can effectively inhibit the growth and reproduction of microorganisms and maintain the freshness of shrimp. On the 8th day of storage, the TVC of the control, which was $6.3 \log _{10} \mathrm{CFU} / \mathrm{g}$, had exceeded the safety limit of $6.0 \log _{10} \mathrm{CFU} / \mathrm{g}$ for pollution-free aquatic 


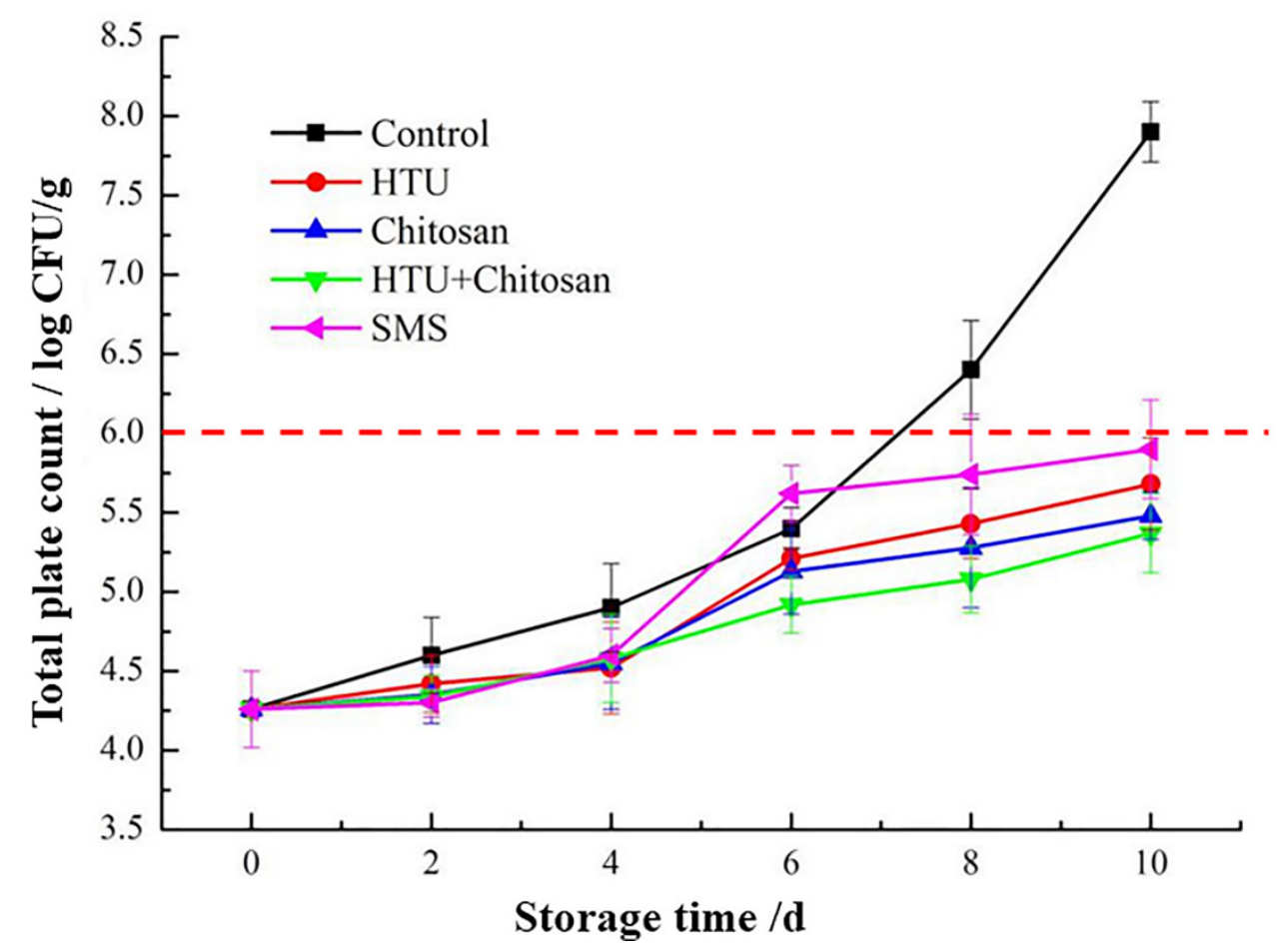

Fig. 1. The total plate count (TVC) changes in Litopenaeus vannamei treated with the different coating solution. The red line $\left(6.0 \log _{10}\right.$ CFU/g) represented the safety limit for TVC in aquatic products. Control, immersed in distilled water; HTU, immersed in 2\% hypotaurine solution; Chitosan, immersed in $1 \%$ chitosan solution; HTU + chitosan, immersed in $2 \%$ hypotaurine solution combined with $1 \%$ chitosan solution; SMS, immersed in $1.25 \%$ sodium metabisulphite.

products (Odeyemi et al., 2020). While at the end of the 10th day, the TVC of shrimp with treatment under $1.25 \%$ SMS, $2 \%$ hypotaurine, $1 \%$ chitosan, and combined with hypotaurine and chitosan coating solution were 5.83, 5.66, 5.42, and 5.25 $\log _{10}$ $\mathrm{CFU} / \mathrm{g}$, respectively. Furthermore, the hypotaurine-chitosan treated shrimp were observed to maintain the lowest TVC value during the whole storage time. The results suggested that coating of chitosan with hypotaurine could have a synergistic effect on microbial activity and a further improvement in antimicrobial properties during frozen storage.

Chitosan-based materials have been emerging as promising and effective bacteriostatic agents for most food-borne pathogens (Rashki et al., 2021), which could retard the growth of spoilage organisms and extend the shelf-life of fish, meat, vegetables and fruits products (Kumar et al., 2020). Ju et al. (2021) also reported that using amino acid modified chitosan showed higher antibacterial efficiency against Escherichia coli than a single chitosan solution, which is agreed with our current study results. The most acceptable proposed mechanism responsible for antimicrobial activities of chitosan is based on the interaction of negatively charged microbial cell membranes with positively charged amine groups in chitosan, destroying the membrane and changing the cell permeability, leading to the leakage of intracellular contents, and ultimately reducing the microbial growth (Hosseinnejad \& Jafari, 2016). In addition, the destruction of the lipopolysaccharide layer of the gram-negative bacteria cell membrane, against by chitosan, can also influence antimicrobial action and lead to cell death (Yuan et al., 2016).

\section{Analysis of $\mathrm{pH}$ value change}

Effect of hypotaurine, chitosan and SMS coating treatments on $\mathrm{pH}$ values of shrimp are shown in Fig. 2(A). The $\mathrm{pH}$ of fresh shrimp at the beginning of storage (Day 0 ) was 7.17. The $\mathrm{pH}$ value in all treatment groups increased significantly $(p<0.05)$ during storage, while the $\mathrm{pH}$ changing rates were varied with each other. Among them, the $\mathrm{pH}$ value of the control group has the largest change compared to other groups $(p<0.05)$. The $\mathrm{pH}$ change in shrimp can be attributed to the accumulation of alkaline compounds caused by lipid peroxidation and protein alteration by various bacterial activities or metabolic enzymes 
(A)

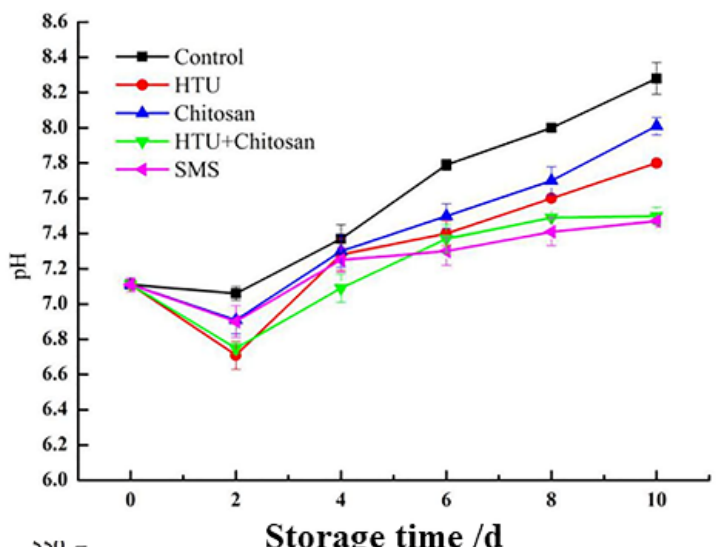

(C)

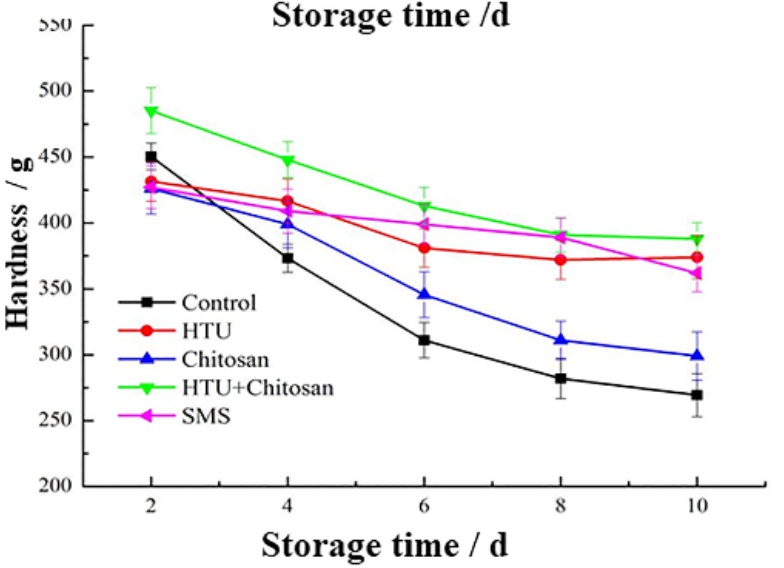

(B)

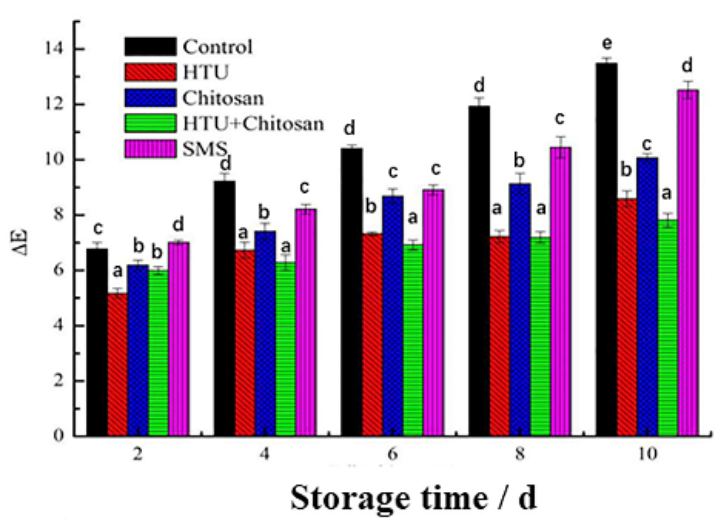

(D)

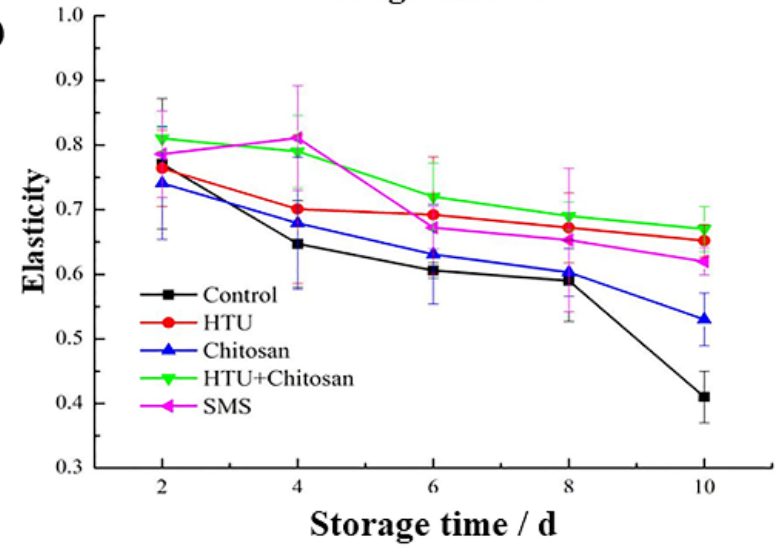

Fig. 2. Physical properties (A) pH value, (B) $\Delta \mathrm{E},(\mathrm{C})$ hardness property, (D) elasticity in Litopenaeus vannamei treated with the different coating solution. ${ }^{\mathrm{a}-\mathrm{e}}$ The various lowercase letters on the same storage time indicated the significant differences $(p<$ 0.05) in $\triangle E$ values under different treatments. Control, distilled water; HTU, hypotaurine solution; Chitosan, chitosan solution; HTU + chitosan, hypotaurine combined with chitosan solution; SMS, sodium metabisulphite.

(Wang et al., 2015). Results obtained from the study showed that shrimp samples both coasted with chitosan alone and hypotaurine-chitosan blending solution can delay the $\mathrm{pH}$ values increase, indicating that the combination of chitosan coating and hypotaurine may reduce the decomposition of protein and improve food safety.

\section{Analysis of color difference}

The $\Delta E$ value was used to measure the color change and comprehensively indict the correlation of color saturation between different treatment groups. Hence, it has been widely accepted as a quality parameter to evaluate food deterioration during storage, especially in seafood (Moradi et al., 2019). As shown in Fig. 2(B), the changes in $\Delta E$ value of shrimp among all treatments increased with the extension of storage time. However, the $\Delta E$ of shrimp coated with chitosan, hypotaurine, and hypotaurine-chitosan were all significantly lower than that of the control group within the 10 days $(p<0.05)$. The lower $\Delta E$ values observed from chitosan, hypotaurine, and hypotaurine-chitosan group suggested that they could effectively delay the color change of shrimp. Particularly, compared with the single hypotaurine or chitosan coating group, the hypotaurine-chitosan blending coating group has the lowest increment in $\Delta \mathrm{E}$ value, indicating that the mixed solution can obviously delay the progress of spoilage and the blackening of shrimp heads. This phenomenon was supposed due to the hypotaurine-chitosan mixed solution contributing to the free-radical scavenging ability and chelation of metal ions (Sathe et al., 2016), and endowing the blend coating with antioxidant activities and enzyme inhibition ability. According to a similar study, $\mathrm{Na}$ et al. (2018) investigated the influence of chitosan $+\varepsilon$-polylysine coating material on pacific white shrimp's sensorial properties. The results showed that the samples treated with chitosan and chitosan with $\varepsilon$-polylysine had higher overall acceptable scores and less color differ- 
ence than the control group during froze storage.

However, during the latter chilled storage period, a marked increase of $\Delta E$ value was noticed, and a peak above 12.5 was achieved at the end of the 10-days storage. This result may be attributed to the fact that the addition of SMS has little influence on preventing the protein denaturation, chemical autolytic reaction and enzyme catalyzed reaction of shrimp during long term storage (Miraglia et al., 2020).

\section{Analysis of textural properties change}

The hardness and elasticity textural properties are two of the most primary qualities to meet the acceptableness of food to consumers. According to the report of Mohan et al. (2012), hardness means the resistance at maximum compression during the first and second compression, while elasticity represents the ability to recover to original form after the deforming force is removed. As can be seen in Fig. 2(C) and (D), the changes in hardness and elasticity values of Litopenaeus vannamei during $4^{\circ} \mathrm{C}$ storage within $10 \mathrm{~d}$ were monitored and recorded. The initial hardness of fresh shrimp was $521 \mathrm{~g}$ (Day 0). A mildly decreasing trend of hardness was shown at the first 2-days, ranging from a control group of $480 \mathrm{~g}$ to the hypotaurine-chitosan group of $495 \mathrm{~g}$. However, thereafter, the hardness parameter of all the shrimp treatment groups decreased significantly $(p<$ 0.05 ), and the hypotaurine-chitosan coating treatment group showed the lowest decreasing trend compared to other treated and untreated samples. The maintain of high hardness (393 $\mathrm{g}$ at 10-day) in hypotaurine-chitosan group could be attributed to the chitosan and hypotaurine mixing coating may function as a proteolysis-resistance agent, thus protein denaturation could be delayed (Salari et al., 2018).

A similar decreasing trend was noticed between elasticity and hardness. The initial elasticity of shrimp was 0.85 , and at the end of the low-temperature storage, the elasticity value of hypotaurine-chitosan, hypotaurine, SMS, chitosan, and control group were $0.69,0.67,0.63,0.54$, and 0.41 , respectively. The results revealed that additional chitosan coating after hypotaurine treatment significantly improved the coating efficiency to Litopenaeus vannamei, and provided protection against the deforming force and enhanced elasticity capacity.

In fact, the textural parameters of shrimp could be affected by various factors. Among them, the stability of myofibrillar protein and the strength of connective tissue played the most important roles (Cao et al., 2019). In this study, these textural results firmly demonstrated the positive synergistic effect of hypotaurine-chitosan coating in textural properties for shrimp during storage. Terriente-Palacios \& Castellari (2022) found that hypotaurine, as one kind of uncommon bioactive molecule, showed potent anti-heavy metals activities. In addition, Colovic et al. (2018) also found it could be demonstrated to provide protection against free radicals. Hence, the simultaneous treatment between chitosan and hypotaurine can retard the denaturation and degradation of myofibrillar protein and collagen proteins, improving the textural properties of shrimp.

\section{Analysis of total volatile base nitrogen (TVB-N) changes}

The main spoilage mechanisms of protein-rich foods, including meat and fish, were occurred by metabolic processes and microbial activity. During the spoilage period, the degradation of proteins and other nitrogen compounds can cause the accumulation of organic amines volatile (Bekhit et al., 2021), such as ammonia, methylamine, dimethylamine, trimethylamine and other similar compounds, which are called TVB-N. Since the TVB-N content always showed a similar trend to other biomarkers of spoilage, it has been widely accepted as a quality indicator of food freshness, especially in seafood. The TVB-N content of shrimp exposed to different treatments during the chilled storage was shown in Fig. 3(A). The control group without any coating treatment of shrimp exhibited a remarkable increase from $3.1 \mathrm{mg} / 100 \mathrm{~g}$ to $41.7 \mathrm{mg} / 100 \mathrm{~g}$ with prolonged storage time during 10 days, and surpassed the threshold of $30.0 \mathrm{mg} / 100 \mathrm{~g}$ at 6 th day after storage. In line with the upward trend of the control group, the TVB-N content of shrimp treated by chitosan, hypotaurine and SMS coating also increased significantly $(p<0.05)$. However, the lowest increase rate was observed in hypotaurine-chitosan coating group, and the TVB-N level was $22.5 \mathrm{mg} / 100 \mathrm{~g}$ at the end of storage, indicating it was still edible and acceptable. Based on the results obtained above, it can be concluded that coating of the hypotaurine and chitosan mixing has a positive effect on shrimp spoilage and protein degradation, thus prolonging the shelf-life of refrigerated shrimp. Similar results have been reported by Zhang et al. (2012) in grass carp with chitosan-antioxidants coating, and the result showed that the TVB-N value in chitosan + acetic acid + tea polyphenol group was significantly less than the control samples $(10.02<22.36 \mathrm{mg} / 100 \mathrm{~g}$, on the 10th day).

The mechanism of the TVB-N accumulation inhibition by chitosan and hypotaurine was supposed. On the one hand, chitosan can alter the microbial cell membranes barrier properties and inhibit their biological activity (Pan et al., 2015), thus 
(A)

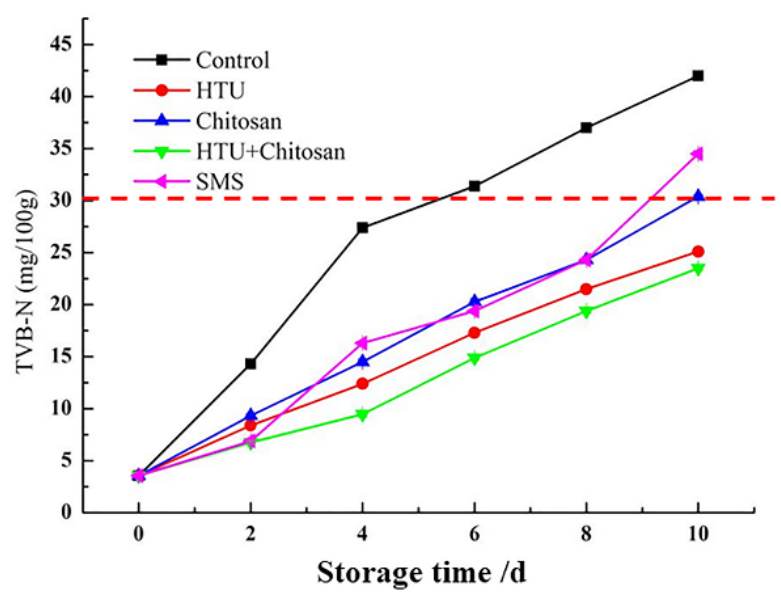

(B)

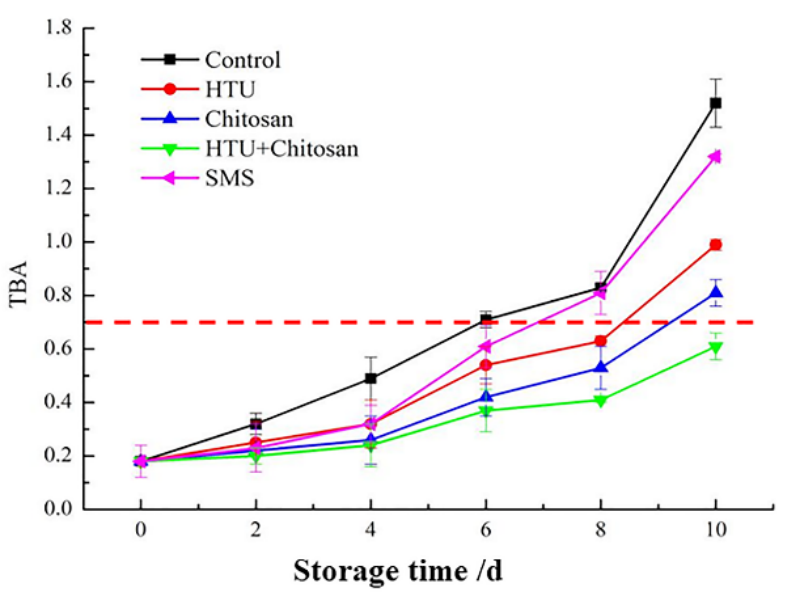

Fig. 3. Freshness indicator of (A) TVB-N and (B) TBA. (A) TVB-N and (B) TBA changes in Litopenaeus vannamei treated with the different coating solution. The red line in TVB-N $(30.0 \mathrm{mg} / 100 \mathrm{~g})$ represented the freshness threshold of nitrogen-compounds accumulation, and in TBA ( $0.7 \mathrm{mg} \mathrm{MDA} / \mathrm{kg}$ ) meant the threshold of lipid oxidation in aquatic products. Control, distilled water; HTU, hypotaurine solution; Chitosan, chitosan solution; HTU + chitosan, hypotaurine combined with chitosan solution; SMS, sodium metabisulphite; TVB-N, total volatile base nitrogen; TBA, thiobarbituric acid.

slowing down the protein decomposition by micro-bacteria in shrimp during storage. On the other hand, hypotaurine, a kind of sulfur-containing amino acid, may play an important role against oxygen reactive species and defense the oxidation of protein and other nitrogen compounds (Métayer et al., 2008).

\section{Thiobarbituric acid (TBA) analysis}

The development of lipid oxidation can be followed by the primary products of lipid oxidation, and secondary oxidation products (Mariutti \& Bragagnolo, 2017). The TBA, a secondary product of oxidative degradation of unsaturated fatty acids, has been widely used for the measurement of malonaldehyde content, and shows the degree of lipid oxidation sate through colorimetric method, particularly in high-lipid seafood (Kulawik et al., 2018).

The influence of different coating treatments on lipid oxidation in shrimp during cold storage was presented in Fig. 3(B). At the beginning of the storage, the TBA value of fresh shrimp was in the range of $0.18-0.20 \mathrm{mg} \mathrm{MDA} / \mathrm{kg}$. During the storage period, the TBA values of all treatment groups were noted with a significant $(p<0.05)$ increment, indicting an accelerated process of the degradation of lipid molecules and formation of secondary oxidation products. Among all samples, the highest increase throughout the chilled storage was measured for the control group (from 0.19 to $1.52 \mathrm{mg} \mathrm{MDA} / \mathrm{kg}$ ), and the result indicated that the control group shrimp was no longer suitable for consumption and testing after six days at $4{ }^{\circ} \mathrm{C}$ storage, because its TBA content was above $0.7 \mathrm{mg} \mathrm{MDA} / \mathrm{kg}$. Shrimp treated with a mixed solution by hypotaurine and chitosan showed the lowest level of TBA, compared to the single solution of $1.25 \%$ SMS, $2 \%$ hypotaurine and $1 \%$ chitosan, during whole the storage at $4{ }^{\circ} \mathrm{C}$ for 10 days $(0.58 \mathrm{mg} \mathrm{MDA} / \mathrm{kg}<0.7 \mathrm{mg} \mathrm{MDA} / \mathrm{kg})$. Hence, it can be concluded that hypotaurine-chitosan coating was effective in controlling lipid oxidation and prolonging the shelf-life during chilled storage of Litopenaeus vannamei.

Shoveler (2005) pointed out that amino acids of taurine and hypotaurine possess the potency to interrupt redox status by reacting with weak $\mathrm{O}-\mathrm{H}$ bonds and alkyl radicals, and thus have an important role in defense against oxidative stress. The blending result of chitosan and hypotaurine coating in shrimp storage showed an improved antioxidation property than the hypotaurine group, confirming the existence of the synergistic effect of the compound solution method.

\section{Analysis of melanosis}

The melanosis scores of Litopenaeus vannamei shrimp under different coating treatments were depicted in Fig. 4. The melanosis scores of all treatment groups were found to increase significantly $(p<0.05)$, indicating that the visual sensory quality of shrimp decreased rapidly along with chilled storage. However, 


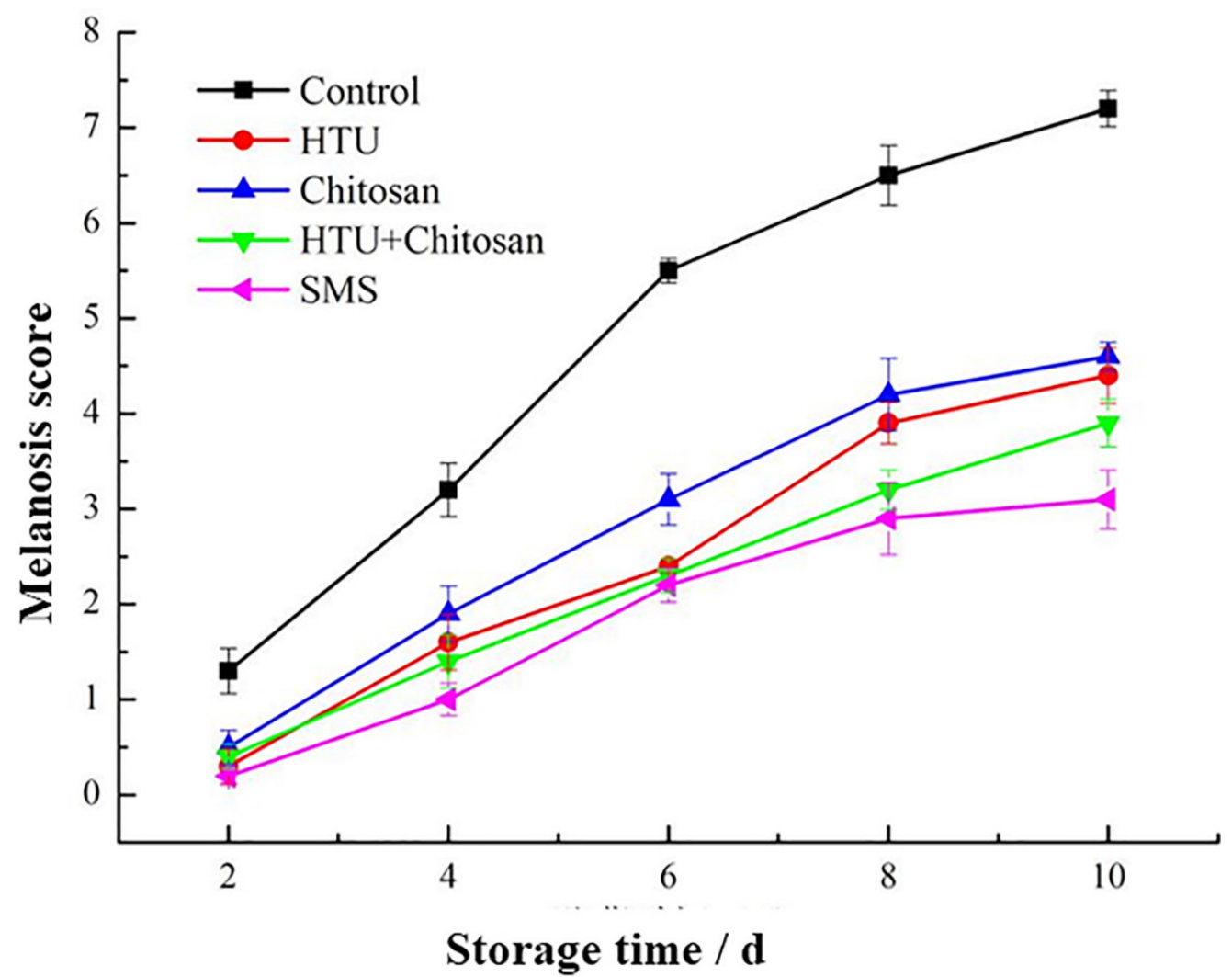

Fig. 4. Melanosis changes in Litopenaeus vannamei treated with the different coating solution. Control, distilled water; HTU, hypotaurine solution; Chitosan, chitosan solution; HTU + chitosan, hypotaurine combined with chitosan solution; SMS, sodium metabisulphite.

the melanosis scores at the end of storage were varied among different treatments, from highest to lowest as follows: the control group (7.2), the single chitosan coating group (4.5), the single hypotaurine coating group (4.4, not significantly different with chitosan group), the hypotaurine-chitosan group (3.6), and the SMS group (2.8). As previously reported, both chitosan and hypotaurine coating plays an effective role to delay the melanosis phenomenon of shrimp during storage. Notably, the lowest scores were observed in shrimp coated with $1.25 \%$ SMS solution, which means that SMS may have the best effective melanosis inhibition ability in this study.

The melanosis in shrimp was mainly occurred by tyrosine converted into quinones by polyphenol oxidase (PPO), and further increase the extent of melanosis (Sae-leaw et al., 2018). Mol and Turkmen (Mol \& Türkmen, 2010) have reported significant improvements in anti-molanosis by SMS, due to preventing enzymatic browning and decreasing dehydration in shrimp. However, the SMS solution may tend to cause sulfur dioxide residues in food and compromise the food safety attributes of the treated shrimp (Thepnuan et al., 2008). Therefore, the hypotaurine combined with chitosan coating could be used as a green and alternative method to alleviate the melanosis of Litopenaeus vannamei during chilled storage.

\section{Sensory evaluation}

Sensory evaluation of shrimp with different coating methods during storage was shown in Fig. 5. In the early four days, the appearance, odor and texture of shrimp with coating treatments were all above 8 scores, better than the control group. The radar chart indicated that the coating methods, with SMS, hypotaurine, chitosan, and mixed hypotaurine-chitosan solution, all have a positive effect for shrimp to maintain the good sensory properties in the early chilled storage. But the sensory evaluation scores show a downward trend with the increase of storage time. Compared with the single coating solution by hypotaurine, chitosan, SMS group, the hypotaurine-chitosan group 

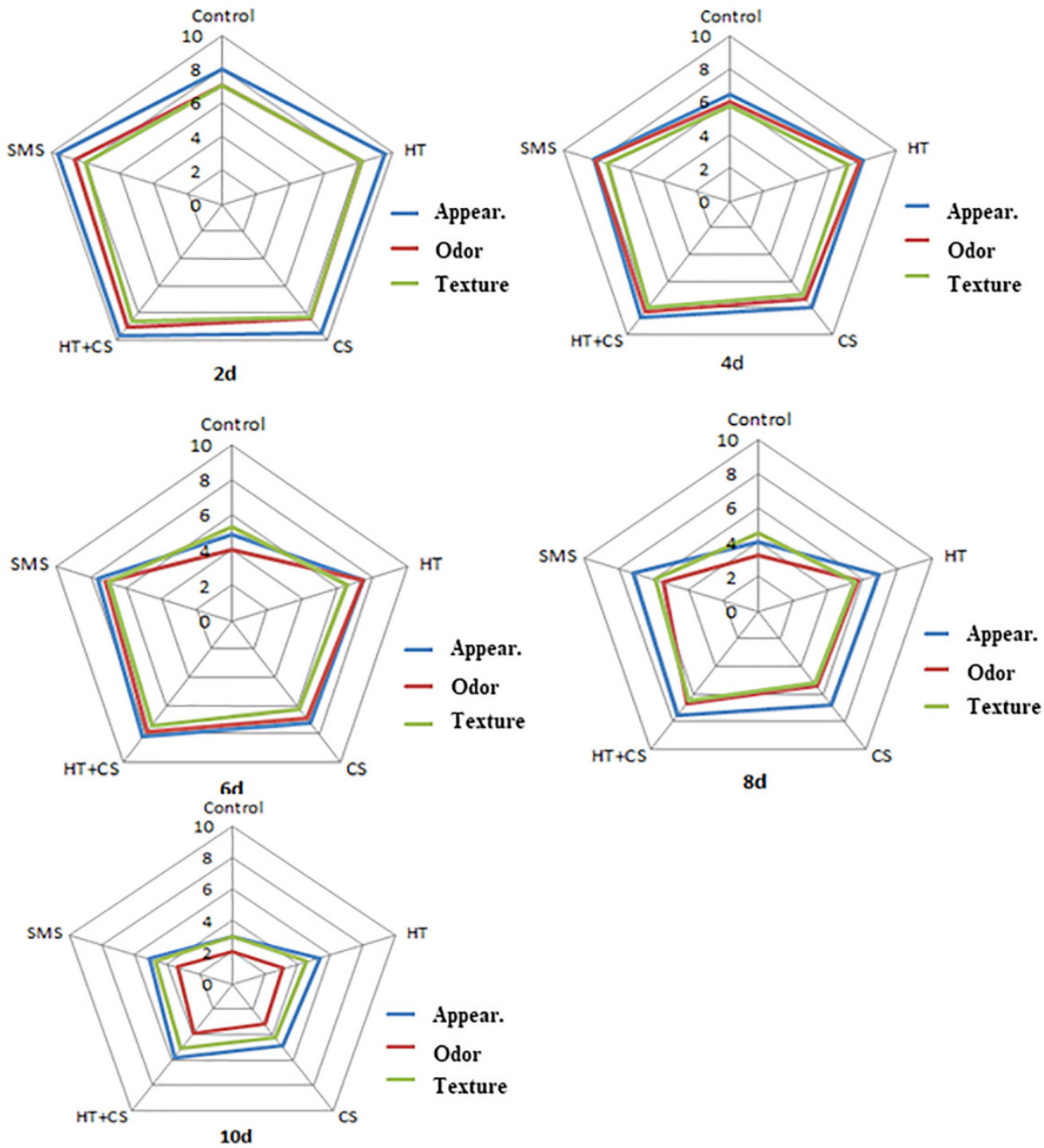

Fig. 5. Sensory evaluation in Litopenaeus vannamei treated with the different coating solution. Control, distilled water; HTU, hypotaurine solution; Chitosan, chitosan solution; HTU + chitosan, hypotaurine combined with chitosan solution; SMS, sodium metabisulphite.

could delay the deterioration of shrimp and prolong the food shelf-life. Similarly, Cheng et al. (2021) also reported that chitosan-based coatings combined with amino-acid could inhibit endogenous protease and show effectively maintain the sensory property of beef slices on chilled storage.

\section{Conclusion}

The present study verified that the blending coating treatment combined with hypotaurine and chitosan served as an effective method to inhibit microbial growth, reduce $\mathrm{pH}$ value and color difference change, and improve the texture properties of hardness and elastic of Litopenaeus vannamei under chilled storage within 10 days. The chitosan coating combined with hypotaurine also showed an excellent performance in controlling protein degradation and lipid oxidation, and decreasing the content of TVB-N and TBA for shrimp. Furthermore, this method can inhibit the degree of melanosis phenomenon and improve the sensory scores of shrimp. These results implied that the chitosan coating combined with hypotaurine can be valuable as a coating 
material for shrimp during storage, with various advantages of ensuring safety quality and prolonging the shelf life of the seafood.

\section{Competing interests}

No potential conflict of interest relevant to this article was reported.

\section{Funding sources}

This work was financially supported by the foundation of $\mathrm{Na}$ tional Natural Science Foundation of China (Foundation of NSFC31871868),Hainan Province Natural Science Foundation of China (321CXTD1012), and Scientific Research Foundation of Hainan Tropical Ocean University (RHDRC202117).

\section{Acknowledgements}

Not applicable.

\section{Availability of data and materials}

Upon reasonable request, the datasets of this study can be available from the corresponding author.

\section{Ethics approval and consent to participate}

This article does not require IRB/IACUC approval because there are no human and animal participants.

\section{ORCID}

Meiyu Chen

Lingping $\mathrm{Hu}$

Zhiheng $\mathrm{Hu}$

Gaoshang Li

Yaoxian Chin

Yaqin $\mathrm{Hu}$

\section{References}

Acharya, M, Lau-Cam, CA. Comparative evaluation of the effects of taurine and thiotaurine on alterations of the cellular redox status and activities of antioxidant and glutathione-related enzymes by acetaminophen in the rat. In: El Idrissi A, LAAmoreaux WJ, editors. Taurine 8. New York: Springer; 2013.

Amparyup P, Charoensapsri W, Tassanakajon A. Prophenoloxidase system and its role in shrimp immune responses against major pathogens. Fish Shellfish Immunol.
2013;34:990-1001.

Bekhit AEDA, Holman BWB, Giteru SG, Hopkins DL. Total volatile basic nitrogen (TVB-N) and its role in meat spoilage: a review. Trends Food Sci Technol. 2021;109:280-302.

Cao H, Zhong S, Zhang B, Wei W, Shen C, Ying X. Sodium erythorbate, stable chlorine dioxide, and gellan gum glazing for shelf life extension of commercial peeled shrimp ( $\mathrm{Li}$ topenaeus vannamei) during frozen storage. J Food Process Preserv. 2019;43:e14108.

Chen M, Yan T, Huang J, Zhou Y, Hu Y. Fabrication of halochromic smart films by immobilizing red cabbage anthocyanins into chitosan/oxidized-chitin nanocrystals composites for real-time hairtail and shrimp freshness monitoring. Int J Biol Macromol. 2021;179:90-100.

Cheng Y, Hu J, Wu S. Chitosan based coatings extend the shelflife of beef slices during refrigerated storage. LWT-Food Sci Technol. 2021:138:110694.

Colovic MB, Vasic VM, Djuric DM, Krstic DZ. Sulphur-containing amino acids: protective role against free radicals and heavy metals. Curr Med Chem. 2018;25:324-35.

Ge Y, Li Y, Bai Y, Yuan C, Wu C, Hu Y. Intelligent gelatin/oxidized chitin nanocrystals nanocomposite films containing black rice bran anthocyanins for fish freshness monitorings. Int J Biol Macromol. 2020;155:1296-306.

Hosseinnejad M, Jafari SM. Evaluation of different factors affecting antimicrobial properties of chitosan. Int J Biol Macromol. 2016;85:467-75.

Ju X, Tian L, Duan X, Li Z, Han Y, Tian Y, et al. Amino acid dependent performance of modified chitosan against bacteria and red blood cell. J Nanosci Nanotechnol. 2021;21:5443-8.

Kulawik P, Alvarez C, Cullen PJ, Aznar-Roca R, Mullen AM, Tiwari B. The effect of non-thermal plasma on the lipid oxidation and microbiological quality of sushi. Innov Food Sci Emerg Technol. 2018;45:412-7.

Kumar S, Mukherjee A, Dutta J. Chitosan based nanocomposite films and coatings: emerging antimicrobial food packaging alternatives. Trends Food Sci Technol. 2020;97:196-209.

Li W, Li Z, Peng MJ, Zhang X, Chen Y, Yang YY, et al. Oenothein B boosts antioxidant capacity and supports metabolic pathways that regulate antioxidant defense in Caenorhabditis elegans. Food Funct. 2020a;11:9157-67.

Li DY, Liu ZQ, Liu B, Qi Y, Liu YX, Liu XY, et al. Effect of protein oxidation and degradation on texture deterioration of ready-to-eat shrimps during storage. J Food Sci. 2020b;85:2673-80. 
Li M, Wang W, Fang W, Li Y. Inhibitory effects of chitosan coating combined with organic acids on Listeria monocytogenes in refrigerated ready-to-eat shrimps. J Food Prot. 2013;76:1377-83.

Lim C, Hwang DS, Lee DW. Intermolecular interactions of chitosan: degree of acetylation and molecular weight. Carbohydr Polym. 2021;259:117782.

Mariutti LRB, Bragagnolo N. Influence of salt on lipid oxidation in meat and seafood products: a review. Food Res Int. 2017;94:90-100.

Métayer S, Seiliez I, Collin A, Duchêne S, Mercier Y, Geraert PA, et al. Mechanisms through which sulfur amino acids control protein metabolism and oxidative status. J Nutr Biochem. 2008;19:207-15.

Miraglia D, Castrica M, Menchetti L, Esposto S, Branciari R, Ranucci D, et al. Effect of an olive vegetation water phenolic extract on the physico-chemical, microbiological and sensory traits of shrimp (Parapenaeus longirostris) during the shelf-life. Foods. 2020;9:1647.

Mohan CO, Ravishankar CN, Lalitha KV, Gopal TKS. Effect of chitosan edible coating on the quality of double filleted Indian oil sardine (Sardinella longiceps) during chilled storage. Food Hydrocoll. 2012;26:167-74.

Montero P, Lopez-Caballero ME, Perez-Mateos M. The effect of inhibitors and high pressure treatment to prevent melanosis and microbial growth on chilled prawns (Penaeus japonicus). J Food Sci. 2001;66:1201-6.

Mol S, Türkmen ÖA. Effect of sodium metabisulfite and citric acid on the quality of crayfish (Astacus leptodactylus). J Muscle Foods. 2010;21:327-42.

Moradi M, Tajik H, Almasi H, Forough M, Ezati P. A novel $\mathrm{pH}$-sensing indicator based on bacterial cellulose nanofibers and black carrot anthocyanins for monitoring fish freshness. Carbohydr Polym. 2019;222:115030.

Na S, Kim JH, Jang HJ, Park HJ, Oh SW. Shelf life extension of Pacific white shrimp (Litopenaeus vannamei) using chitosan and $\varepsilon$-polylysine during cold storage. Int J Biol Macromol. 2018;115:1103-8.

Negm NA, Hefni HHH, Abd-Elaal AAA, Badr EA, Abou Kana MTH. Advancement on modification of chitosan biopolymer and its potential applications. Int J Biol Macromol. 2020;152:681-702.

Odeyemi OA, Alegbeleye OO, Strateva M, Stratev D. Understanding spoilage microbial community and spoilage mechanisms in foods of animal origin. Compr Rev Food
Sci Food Saf. 2020;19:311-31.

Pan Y, Huang X, Shi X, Zhan Y, Fan G, Pan S, et al. Antimicrobial application of nanofibrous mats self-assembled with quaternized chitosan and soy protein isolate. Carbohydr Polym. 2015;133:229-35.

Rashki S, Asgarpour K, Tarrahimofrad H, Hashemipour M, Ebrahimi MS, Fathizadeh H, et al. Chitosan-based nanoparticles against bacterial infections. Carbohydr Polym. 2021;251:117108.

Sae-leaw T, Benjakul S, Vongkamjan K. Retardation of melanosis and quality loss of pre-cooked Pacific white shrimp using epigallocatechin gallate with the aid of ultrasound. Food Control. 2018;84:75-82.

Salari M, Khiabani MS, Mokarram RR, Ghanbarzadeh B, Kafil HS. Development and evaluation of chitosan based active nanocomposite films containing bacterial cellulose nanocrystals and silver nanoparticles. Food Hydrocoll. 2018;84:414-23.

Sathe P, Richter J, Myint MTZ, Dobretsov S, Dutta J. Self-decontaminating photocatalytic zinc oxide nanorod coatings for prevention of marine microfouling: a mesocosm study. Biofouling. 2016;32:383-95.

Sánchez-Muros MJ, Renteria P, Vizcaino A, Barroso FG. Innovative protein sources in shrimp (Litopenaeus vannamei) feeding. Rev Aquacult. 2020;12:186-203.

Sharifian S, Shabanpour B, Taheri A, Kordjazi M. Effect of phlorotannins on melanosis and quality changes of Pacific white shrimp (Litopenaeus vannamei) during iced storage. Food Chem. 2019;298:124980.

Shiekh KA, Benjakul S, Sae-Leaw T. Effect of Chamuang (Garcinia cowa Roxb.) leaf extract on inhibition of melanosis and quality changes of Pacific white shrimp during refrigerated storage. Food Chem. 2019;270:554-61.

Shoveller AK, Stoll B, Ball RO, Burrin DG. Nutritional and functional importance of intestinal sulfur amino acid metabolism. J Nutr. 2005;135:1609-12.

Terriente-Palacios C, Castellari M. Levels of taurine, hypotaurine and homotaurine, and amino acids profiles in selected commercial seaweeds, microalgae, and algae-enriched food products. Food Chem. 2022;368:130770.

Thepnuan R, Benjakul S, Visessanguan W. Effect of pyrophosphate and 4-hexylresorcinol pretreatment on quality of refrigerated white shrimp (Litopenaeus vannamei) kept under modified atmosphere packaging. J Food Sci. 2008;73:S12433. 
Wang Y, Liu L, Zhou J, Ruan X, Lin J, Fu L. Effect of chitosan nanoparticle coatings on the quality changes of postharvest whiteleg shrimp, Litopenaeus vannamei, during storage at $4{ }^{\circ} \mathrm{C}$. Food Bioprocess Technol. 2015;8:907-15.

Wu C, Hu Y, Chen S, Chen J, Liu D, Ye X. Formation mechanism of nano-scale antibiotic and its preservation performance for silvery pomfret. Food Control. 2016;69:331-8.

Yuan G, Chen X, Li D. Chitosan films and coatings containing essential oils: the antioxidant and antimicrobial activity, and application in food systems. Food Res Int. 2016;89:11728.

Zhang L, Luo Y, Hu S, Shen H. Effects of chitosan coatings enriched with different antioxidants on preservation of grass carp (Ctenopharyngodon idellus) during cold storage. J Aquat Food Prod Technol. 2012;21;508-18. 